\title{
Late Neonatal Death
}

National Cancer Institute

\section{Source}

National Cancer Institute. Late Neonatal Death. NCI Thesaurus. Code C116773.

Death of live newborn between 7 and 27 days after birth. 